\title{
Pseudoclassical model for Weyl particle in 10 dimensions
}

\author{
D. M. Gitman, and A. E. Gonçalves. \\ Instituto de Física, Universidade de São Paulo \\ P.O. Box 66318, 05389-970 São Paulo, SP, Brazil
}

(October 19, 2018)

\begin{abstract}
A pseudoclassical model to describe Weyl particle in 10 dimensions is proposed. In course of quantization both the massless Dirac equation and the Weyl condition are reproduced automatically. The construction can be relevant to Ramond-Neveu-Schwarz strings where the Weyl reduction in the Ramond sector has to be made by hands.
\end{abstract}

PACS number(s): 11.10.Ef, 036.65.Pm

Typeset using REVTEX 
Classical and pseudoclassical models of relativistic particles and their quantization attract attention already for a long time. One of the main reason to study them is related to the string theory, because point-like particles can be treated as prototypes of strings or some modes in string theory. Recently [1], a pseudoclassical model to describe Weyl particle in 4 dimensions was proposed. The limit $m \rightarrow 0$ of the standard action of a spinning particle [2] in 4 dimensions was modified essentially to get the minimal theory (Weyl theory) of a massless spinning particle. Thus, both Dirac equation and Weyl condition are reproduced in course of a quantization. It turns out to be possible to adopt the model to 10 dimensions. That is important in connection with superstring theory problems in such dimensions [3], where, for example, the minimal quantum theory of Ramond-Neveu-Schwarz string does not appear automatically, and the corresponding GSO reduction (in particular Weyl reduction of the Ramond sector) has to be made by hands [4].

The action of the Weyl particle in 10 dimensions we are proposing has the form

$$
S=\int_{0}^{1}\left[-\frac{z^{2}}{2 e}-\imath \psi_{\mu} \dot{\psi}^{\mu}\right] d \tau, \quad z^{\mu}=\dot{x}^{\mu}-\imath \psi^{\mu} \chi-\frac{1}{8 !} \epsilon^{\mu \nu \rho_{2} \ldots \rho_{9}} b_{\nu} \psi_{\rho_{2}} \ldots \psi_{\rho_{9}}+\frac{\alpha}{2^{8}} b^{\mu}
$$

where $x^{\mu}, e$ are even and $\psi^{\mu}, \chi$ are odd variables dependent on a parameter $\tau \in[0,1], \mu=$ $\overline{0,9}, \eta_{\mu \nu}=\operatorname{diag}(1,-1, \ldots,-1)$ is Minkowski tensor in 10 dimensions, the variables $b^{\mu}$ form an even 10-vector, and $\alpha$ is an even constant. There are three types of gauge transformations under which the action (1) is invariant: reparametrizations

$$
\delta x^{\mu}=\dot{x}^{\mu} \xi, \quad \delta e=\frac{d}{d \tau}(e \xi), \delta b^{\mu}=\frac{d}{d \tau}\left(b^{\mu} \xi\right), \delta \psi^{\mu}=\dot{\psi}^{\mu} \xi, \quad \delta \chi=\frac{d}{d \tau}(\chi \xi)
$$

with an even parameter $\xi(\tau)$; and two kinds of supertransformations: first ones

$$
\delta x^{\mu}=\imath \psi^{\mu} \epsilon, \quad \delta e=\imath \chi \epsilon, \quad \delta b^{\mu}=0, \delta \psi^{\mu}=\frac{1}{2 e} z^{\mu} \epsilon, \delta \chi=\dot{\epsilon},
$$

with an odd parameter $\epsilon(\tau)$; and second ones

$$
\begin{aligned}
& \delta x^{\mu}=\left(\frac{1}{8 !} \epsilon^{\mu \nu \rho_{2} \ldots \rho_{9}} b_{\nu} \psi_{\rho_{2}} \ldots \psi_{\rho_{9}}-\frac{\alpha}{2^{8}} b^{\mu}\right) \kappa, \delta \psi^{\mu}=\frac{\imath}{8 ! e} \epsilon^{\mu \nu \rho_{2} \ldots \rho_{9}} b_{\nu} z_{\rho_{2}} \psi_{\rho_{3}} \ldots \psi_{\rho_{9}} \kappa, \\
& \delta b^{\mu}=\frac{d}{d \tau}\left(b^{\mu} \kappa\right)-\left(\frac{6}{\alpha}\right) \frac{2^{8}}{8 !} \epsilon^{\mu \nu \rho_{2} \ldots \rho_{9}} \dot{\psi}_{\rho_{2}} \psi_{\rho_{2}} \ldots \psi_{\rho_{9}} \kappa, \delta \chi=0, \quad \delta e=0,
\end{aligned}
$$


with an even parameter $\kappa(\tau)$.

Introducing the canonical momenta

$$
\begin{aligned}
& \pi_{\mu}=\frac{\partial L}{\partial \dot{x}^{\mu}}=-\frac{1}{e} z_{\mu}, P_{e}=\frac{\partial L}{\partial \dot{e}}=0, P_{\chi}=\frac{\partial_{r} L}{\partial \dot{\chi}}=0 \\
& P_{\mu}=\frac{\partial_{r} L}{\partial \dot{\psi}^{\mu}}=-\imath \psi_{\mu}, P_{b_{\mu}}=\frac{\partial L}{\partial \dot{b}^{\mu}}=0
\end{aligned}
$$

we discover primary constraints $\Phi^{(1)}=0\left(\Phi_{1}^{(1)}=P_{e}, \Phi_{2}^{(1)}=P_{\chi}, \Phi_{3 \mu}^{(1)}=P_{\mu}+\imath \psi_{\mu}, \Phi_{4 \mu}^{(1)}=\right.$ $P_{b_{\mu}}$ ). Then the total Hamiltonian $H^{(1)}$ constructed according to the standard procedure [5.6], has the form $H^{(1)}=H+\lambda_{A} \Phi_{A}^{(1)}$, where

$$
H=-\frac{e}{2} \pi^{2}+\imath \pi_{\mu} \psi^{\mu} \chi-\left(\frac{1}{8 !} \epsilon_{\nu \mu \rho_{2} \ldots \rho_{9}} \pi^{\mu} \psi^{\rho_{2}} \psi^{\rho_{9}}+\frac{\alpha}{2^{8}} \pi_{\nu}\right) b^{\nu}
$$

Using it, one gets secondary constraints $\Phi^{(2)}=0$,

$$
\Phi_{1}^{(2)}=\pi^{2}, \quad \Phi_{2}^{(2)}=\pi_{\mu} \psi^{\mu}, \quad \Phi_{3 \mu}^{(2)}=\frac{1}{8 !} \epsilon_{\mu \nu \rho_{2} \ldots \rho_{9}} \pi^{\nu} \psi^{\rho_{2}} \ldots \psi^{\rho_{9}}+\frac{\alpha}{2^{8}} \pi_{\mu}
$$

One can go over from the initial set of constraints $\left(\Phi^{(1)}, \Phi^{(2)}\right)$ to the equivalent one $\left(\Phi^{(1)}, \widetilde{\Phi}^{(2)}\right)$, where $\widetilde{\Phi}^{(2)}=\left.\Phi^{(2)}\right|_{\psi \rightarrow \widetilde{\psi}=\psi+\frac{2}{2} \Phi_{3}^{(1)}}$. The new set of constraints can be explicitly divided in a set of first-class constraints, which is $\left(\Phi_{1,2}^{(1)}, \Phi_{4}^{(1)}, \widetilde{\Phi}^{(2)}\right)$ and in a set of second-class constraints $\Phi_{3}^{(1)}$.

Consider the Dirac quantization, where the second-class constraints $\Phi_{3}^{(1)}$ defines Dirac brackets and therefore the commutation relations, whereas, the first-class constraints, being applied to the state vectors, define physical states [5]. For essential operators and nonzeroth commutators we get:

$$
\left[\hat{x}^{\mu}, \hat{\pi}_{\nu}\right]_{-}=\imath\left\{x^{\mu}, \pi_{\nu}\right\}_{D\left(\Phi_{3}^{(1)}\right)}=\imath \delta_{\nu}^{\mu}, \quad\left[\hat{\psi}^{\mu}, \hat{\psi}^{\nu}\right]_{+}=\imath\left\{\psi^{\mu}, \psi^{\nu}\right\}_{D\left(\Phi_{3}^{(1)}\right)}=-\frac{1}{2} \eta^{\mu \nu}
$$

It is possible to construct a realization of the commutation relation (7) in a Hilbert space $\mathcal{R}$ whose elements $\Psi$ are 32-components columns. Taking into account trivial first-class constraints $\Phi_{1,2,4}^{(1)}$, we can select $\Psi$ dependent only on $x$. Then

$$
\hat{x}^{\mu}=x^{\mu} \mathbf{I}, \quad \hat{\pi}_{\mu}=-\imath \partial_{\mu} \mathbf{I}, \quad \hat{\psi}^{\mu}=\frac{\imath}{2} \gamma^{\mu},
$$


where $\mathbf{I}$ is $32 \times 32$ unit matrix and $\gamma^{\mu}$ are the $\gamma$-matrices in 10 dimensions, $\left[\gamma^{\mu}, \gamma^{\nu}\right]_{+}=2 \eta^{\mu \nu}$. In the realization (8) the operators $\hat{\Phi}^{(2)}$, which corresponds to the first-class constraints (6), have the following form

$$
\hat{\Phi}_{1}^{(2)}=-\partial_{\mu} \partial^{\mu}, \quad \hat{\Phi}_{2}^{(2)}=\frac{1}{2} \partial_{\mu} \gamma^{\mu}, \quad \hat{\Phi}_{3 \mu}^{(2)}=\frac{\imath}{2^{8}}\left[\gamma_{\mu} \gamma^{11} \partial_{\nu} \gamma^{\nu}+\partial_{\mu}\left(\gamma^{11}-\alpha\right)\right]
$$

One can see that conditions $\hat{\Phi}^{(2)} \Psi(x)=0$, are reduced to the following set of independent equations

$$
\partial_{\mu} \gamma^{\mu} \Psi(x)=0, \quad \partial_{\mu}\left(\gamma^{11}-\alpha\right) \Psi(x)=0
$$

The first one is the Dirac equation for massless particles in 10 dimensions and the second one at $\alpha=\mp 1$ is equivalent to Weyl or anti-Weyl conditions $\left(1 \pm \gamma^{11}\right) \Psi(x)=0$ if we consider only normalized functions $\Psi(x)$. Thus, we get automatically projections with positive and negative chirality in course of quantization.

The canonical quantization can be made similar to 4 dimensional case [1] and leads to same quantum mechanics.

\section{ACKNOWLEDGMENTS}

The authors thank N. Berkovits for helpful discussions and Brazilian foundations CNPq and CAPES for support. 


\section{REFERENCES}

[1] D.M. Gitman, A.E. Gonçalves and I.V. Tyutin, Phys. Rev. D 34, 5439 (1994).

[2] F.A. Berezin and M.S. Marinov, JETP Lett. 21, 320 (1975), Ann. Phys. 104, 336 (1977); R. Casalbuoni, Nuovo Cim. A33, 115 (1976); A. Barducci, R. Casalbuoni and L. Lusanna, Nuovo Cim. A35, 377 (1976); L. Brink, S. Deser, B. Zumino, P. Di Vecchia and P. Howe, Phys. Lett. B64, 435 (1976); L. Brink, P. Di Vecchia and P. Howe, Nucl. Phys. B118, 76 (1977); A.P. Balachandran, P. Salomonson, B. Skagerstam and J. Winnberg, Phys. Rev. D15, 2308 (1977).

[3] M.B. Green and J.H. Schwarz, Superstring Theory (Cambridge University Press, Cambridg, 1987).

[4] P. Ramond, Phys. Rev. D 3, 2415 (1971); F. Gliozzi, J. Scherk and D. Olive, Phys. Lett. 65B, 282 (1976).

[5] P.A.M. Dirac, Lectures on Quantum Mechanics (Yeschiva University, 1964).

[6] D.M. Gitman and I.V. Tyutin, Class. Quantum Grav. 7, 2131 (1990); Quantization of Fields with Constraints (Springer, Berlin, 1990). 